\title{
Theoretic quantum Information entropies for the generalized hyperbolic potential
}

\author{
Akpan Ikot ${ }^{1}$, Gaotsiwe Joel Rampho ${ }^{1}$, Precious Amadi ${ }^{2,3}$, Uduakobong Okorie ${ }^{3}$, \\ Makagamathe Sithole ${ }^{1}$, and Mantile Lekala ${ }^{1}$ \\ ${ }^{1}$ University of South Africa \\ ${ }^{2}$ Kirchhoff-Institut für Physik \\ ${ }^{3}$ University of Port Harcourt
}

May 8, 2020

\begin{abstract}
The Shannon entropy (S) and the Fisher Information (I) entropies are investigated for a generalized hyperbolic potential in position and momentum spaces. Firstly, the Schrodinger equation is solved exactly using the Nikiforov-Uvarov-Functional Analysis (NUFA) method to obtain the energy spectra and the corresponding wave function. By Fourier transforming the position space wave function, the corresponding momentum wave function was obtained for the low lying states corresponding to the ground and first excited state. The positions and momentum Shannon entropy and Fisher Information entropies were calculated numerically. Finally, the Bialynicki-Birula and Mycielski (BBM) and the Stam-Cramer-Rao inequalities for the Shannon entropy and Fisher Information entropies respectively were tested and was found to be satisfied for all cases considered
\end{abstract}

\section{Hosted file}

generalized hyperbolic potential.doc available at https://authorea.com/users/291200/articles/ 449293-theoretic-quantum-information-entropies-for-the-generalized-hyperbolic-potential 